\title{
EFFECTS OF LINER GEOMETRY ON ACOUSTIC IMPEDANCE
}

\author{
Michael G. Jones, ${ }^{*}$ Maureen B. Tracy, ${ }^{\dagger}$ Willie R. Watson ${ }^{\ddagger}$ and Tony L. Parrott ${ }^{\S}$ \\ NASA Langley Research Center \\ Hampton, VA
}

\begin{abstract}
$\underline{\text { Abstract }}$
Current aircraft engine nacelles typically contain acoustic liners consisting of perforated sheets bonded onto honeycomb cavities. Numerous models have been developed to predict the acoustic impedance of these liners in the presence of grazing flow, and to use that information with aeroacoustic propagation codes to assess nacelle liner noise suppression. Recent efforts have provided advances in impedance eduction methodologies that offer more accurate determinations of acoustic liner properties in the presence of grazing flow. The current report provides the results of a parametric study, in which a finite element method was used to assess the effects of variations of the following geometric parameters on liner impedance, with and without the presence of grazing flow: percent open area, sheet thickness, sheet thickness-to-hole diameter ratio and cavity depth. Normal incidence acoustic impedances were determined for eight acoustic liners, consisting of punched aluminum facesheets bonded to hexcell honeycomb cavities. Similar liners were tested in the NASA Langley Research Center grazing incidence tube to determine their response in the presence of grazing flow. The resultant data provide a quantitative assessment of the effects of these perforate, single-layer liner parameters on the acoustic impedance of the liner.
\end{abstract}

*Research Scientist, Structural Acoustics Branch, Aerodynamics, Aerothermodynamics and Acoustics Competency, Member of AIAA

${ }^{\dagger}$ Research Scientist, Aeroacoustics Branch, Aerodynamics, Aerothermodynamics and Acoustics Competency

¥Senior Research Scientist, Computational Modeling and Simulation Branch, Aerodynamics, Aerothermodynamics and Acoustics Competency, Member of AIAA

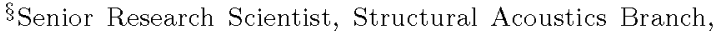
Aerodynamics, Aerothermodynamics and Acoustics Competency

Copyright (C)2002 by the American Institute of Aeronautics and Astronautics, Inc. No copyright is asserted in the United States under Title 17, U.S. Code. The U.S. Government has a royalty-free license to exercise all rights under the copyright claimed herein for government purposes. All other rights are reserved by the copyright owner.

\section{Nomenclature}

$c \quad$ sound speed in duct, $\mathrm{m} / \mathrm{s}$

$d, \tau \quad$ perforate hole diameter and sheet

thickness, mm

D $\quad$ cavity depth, mm

$f \quad$ frequency, $\mathrm{s}^{-1}$

FEM finite element method

GIT grazing incidence tube

$H \quad$ duct height, $\mathrm{m}$

$k$ free space wavenumber, $\mathrm{m}^{-1}$

$L \quad$ FEM computational domain length, $m$

$L_{1}, L_{2}$ distance from source plane to liner leading and trailing edges, $m$

$M \quad$ average Mach number across duct cross-section

NIT normal incidence tube

$p(x, y)$ complex acoustic pressure, $\mathrm{Pa}$

$p_{\text {ref }} \quad$ reference acoustic pressure, $20 \mu \mathrm{Pa}$

$p_{s} \quad$ source plane acoustic pressure, $\mathrm{Pa}$

POA percent open area

SPL sound pressure level, $\mathrm{dB}$

$t$ dimensional time, $\mathrm{s}$

$x, y \quad$ axial and transverse coordinates, $\mathrm{m}$

$x_{i} \quad$ wall measurement location, $\mathrm{m}$

Symbols:

$\delta^{*} \quad$ boundary layer displacement

thickness, mm

$\phi \quad$ measured phase, radians

$\rho_{0} \quad$ ambient density, $\mathrm{kg} / \mathrm{m}^{3}$

$\sigma \quad$ porosity $(P O A / 100)$

$\theta$ normalized acoustic resistance, real component of $\zeta$

$\theta_{\mathrm{RH}} \quad$ normalized acoustic resistance predicted by Rice-Heidelberg model

$\omega \quad$ angular frequency, $\mathrm{s}^{-1}$

$\chi \quad$ normalized acoustic reactance, imaginary component of $\zeta$

$\zeta \quad$ normal incidence acoustic impedance

$(\theta+i \chi)$, normalized by $\rho_{0} c$

$\zeta_{e} \quad$ normalized exit plane acoustic impedance

An $e^{i \omega t}$ time convention will be used throughout this paper. 


\section{Introduction}

The continual increase in commercial aircraft traffic places an increasing emphasis on noise abatement research. One critical component of noise abatement methodology is the use of passive acoustic liners that are integrated into the walls of the engine nacelle inlets and aft-fan ducts. Optimal design of acoustic liners for maximum absorption requires thorough understanding of the effects of liner geometry variations on the acoustic absorption. Such an understanding requires accurate impedance eduction techniques which, when used together with aeroacoustic propagation codes, provide the necessary information to determine the nacelle acoustic liner suppression provided by selected acoustic liner configurations. A recent report ${ }^{1}$ presented a waveguide method that incorporates a finite element technique to educe the acoustic impedance of acoustic liners in the presence of grazing flow.

Impedance prediction models for perforate liners have been investigated over the last five decades. A sampling of the variety of this research can be found in references 2 through 9 . These models have been based on experimental data (see, for example, ref. 10) acquired with a variety of impedance eduction methods. Thus, to properly understand this data set, a thorough understanding of the underlying assumptions in each impedance eduction method is required. To further complicate the issue, there has been a recent interest in the use of perforate liners with geometric parameters that have not been as well studied, especially in the presence of Mach 0.5 grazing flow.

The purpose of this paper is to extend our knowledge of the effects of liner geometry on acoustic impedance for liner configurations consisting of perforated sheets bonded onto honeycomb cavities. In these tests, parametric variations in percent open area, sheet thickness, sheet thickness-to-hole diameter ratio and cavity depth on the acoustic impedance of single-degree-of-freedom liners are investigated. A set of eight liner configurations, consisting of punched aluminum facesheets bonded to hexcell honeycomb cavities, was tested in the NASA Langley Research Center Flow Impedance Test Facility. Specifically, these tests were designed to improve understanding of the effects of high speed grazing flow on low percent open area liners, while covering the typical ranges of perforate hole diameter and sheet thickness. For completeness, test liners with different cavity depths were also included. For each configuration, two test liners were fabricated. The first was designed for testing in a normal incidence tube, which is used to determine the normal incidence acoustic impedance of the test liner in the absence of flow. The second, fabricated using the same material, was designed such that it could be mounted into a grazing incidence tube, which is used to educe the normal incidence acoustic impedance of the test liner in the presence of grazing flow.

The remainder of this paper is organized into four sections. The first section gives a description of the test liners and the waveguides in which they were tested, and also describes the data acquisition system for the two impedance eduction methodologies. The second section provides a brief discussion of the theory underlying the finite element method used to educe the acoustic impedance in the presence of grazing flow. The third section contains a discussion of the results obtained from the data acquired in the NASA Langley Flow Impedance Test Facility. Concluding remarks are presented in the final section.

\section{Experimental Setup}

\section{Description of Test Liners}

Each of the acoustic liners used in this study consisted of a perforated facesheet (punched aluminum) bonded onto a hexcell honeycomb core. Table 1 contains a list of geometric parameters for each of the eight configurations. Two test liners were fabricated for each configuration, with active areas of $51 \mathrm{~mm} \times 51 \mathrm{~mm}$ and $51 \mathrm{~mm} \times 406 \mathrm{~mm}$, respectively. Each was sealed into a container that precluded communication between adjacent cells. The first liner was designed to be mounted onto the end of the NASA Langley Research Center normal incidence tube, with a cross-section of $51 \mathrm{~mm} \times 51 \mathrm{~mm}$. This device was used to determine the normal incidence acoustic impedance of the test liner in the absence of flow. Impedance results acquired with this device have been previously demonstrated to match predicted impedances for calibration liners that could be analyzed from first principles. ${ }^{8}$ The second liner was designed to be mounted into the NASA Langley Research Center grazing incidence tube, which also has a cross-section of $51 \mathrm{~mm} \times 51 \mathrm{~mm}$. This apparatus was used to educe the normal incidence acoustic impedance of the test liner in the presence of grazing flow, for flow rates of $M=0.0,0.1,0.3$ and 0.5 .

Figure 1 contains a sketch of a $51 \mathrm{~mm} \times 406 \mathrm{~mm}$ liner. As indicated in table 1 , the percent open area $(P O A)$, hole diameter $(d)$, facesheet thickness $(\tau)$ and cavity depth $(D)$ ranges are 


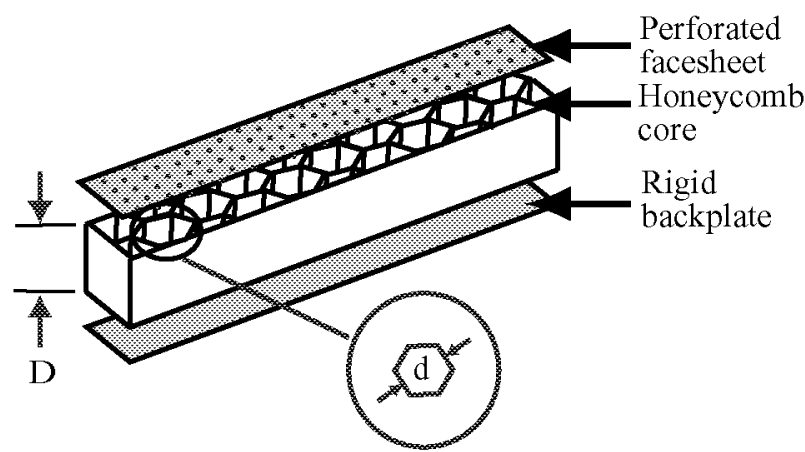

Fig. 1. Sketch of test liner.

$$
\begin{gathered}
6.4 \% \leq P O A \leq 13.2 \% \\
1.0 \mathrm{~mm} \leq d \leq 2.4 \mathrm{~mm} \\
0.5 \mathrm{~mm} \leq \tau \leq 1.0 \mathrm{~mm} \\
38.1 \mathrm{~mm} \leq D \leq 76.2 \mathrm{~mm}
\end{gathered}
$$

These ranges were chosen to be consistent with those used in current aircraft. For completeness, the sheet thickness-to-hole diameter ratio $(\tau / d)$ is also included in the table.

\section{Waveguides and Data Acquisition}

\section{Normal Incidence Tube}

Two waveguides were used in this investigation, both located in the NASA Langley Research Center Flow Impedance Test Facility. One is a normal incidence tube (NIT), used to determine the normal incidence acoustic impedance in the absence of flow. A sketch of the NIT, along with the instrumentation used, is provided in figure 2. The cross-section of the tube is $51 \mathrm{~mm} \times 51 \mathrm{~mm}$, and the length is $584 \mathrm{~mm}$. The test liner is installed in an enclosure that is carefully clamped to the exit plane of the NIT, such that there is no leakage of sound around the edges of the test liner.

Six 120-Watt acoustic drivers generate discretetone acoustic plane waves that impinge upon the surface of the test liner. These acoustic waves are reflected from the liner surface to create a standing wave pattern that uniquely characterizes the normal incidence acoustic impedance of the test liner within its linear regime. A $6.35-\mathrm{mm}$ (1/4-inch) condensertype microphone, flush-mounted in the top wall $6.4 \mathrm{~mm}$ from the face of the test liner, is used as a reference microphone to measure the sound pressure level near the face of the liner. This allows the sound pressure level at the liner face to be held

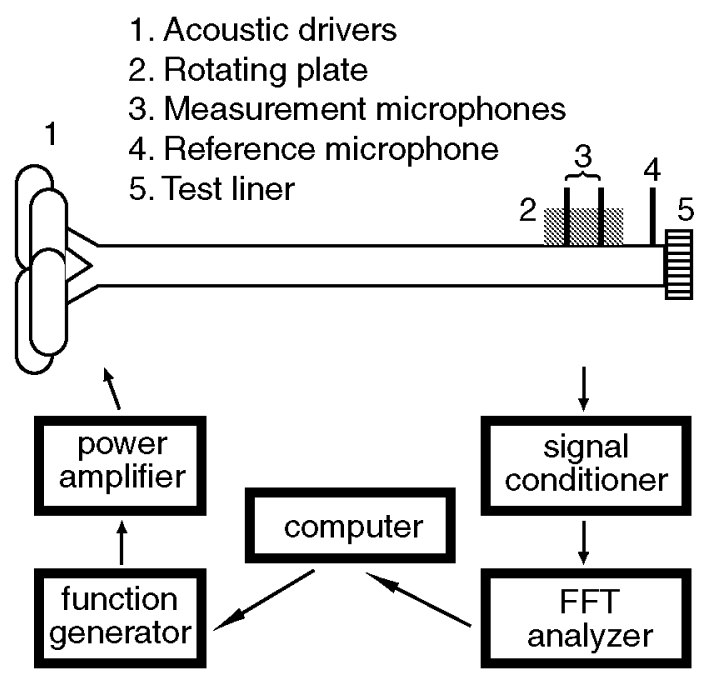

Fig. 2. NASA LaRC Normal Incidence Tube with instrumentation.

constant as the frequency is varied. For the current investigation, data were acquired over the frequency range of 1.0 to $3.0 \mathrm{kHz}$, in steps of $0.5 \mathrm{kHz}$, at a reference level of $120 \mathrm{~dB}$. The NIT also contains a rotating plate that holds two $6.35-\mathrm{mm}$ (1/4-inch) condenser-type microphones. Each microphone is flush mounted into the rotating plate, and the plate is designed such that the two microphone locations can be easily and precisely interchanged by rotating the plate 180 degrees. By taking the average of the two transfer functions between the two microphones in the rotating plate, before and after rotation, differences between the calibration factors (amplitude and phase) of the two microphones are eliminated. The averaged transfer function is then used with the two-microphone method ${ }^{11}$ to educe the acoustic impedance of the test liner.

\section{Grazing Incidence Tube}

The second waveguide allows grazing incidence sound and flow across the liner surface. This grazing incidence tube (GIT) is used to educe the normal incidence acoustic impedance of the test liner in the presence of grazing flow. A schematic of the GIT is provided in figure 3 . This apparatus has a $51 \mathrm{~mm} \times 51 \mathrm{~mm}$ cross-section in which a controlled aeroacoustic environment is achieved. The $51 \mathrm{~mm}-$ wide $\times 406 \mathrm{~mm}$-long test liner is centered in the test section, and is mounted such that its surface forms the lower wall of the GIT.

The desired aeroacoustic environment in the test section is achieved with four 120-Watt acoustic drivers. Their phase-matched outputs are combined 


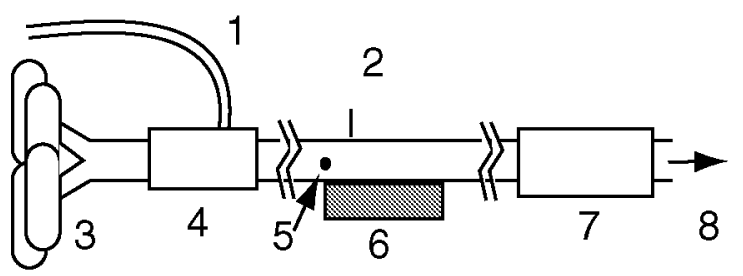

1. High pressure air line 5. Reference microphone 2. Traversing microphone 6 . Test section with liner 3. Acoustic drivers 7. Termination 4. Plenum 8. To vacuum pumps

\section{Fig. 3. NASA LaRC Grazing Incidence Tube.}

to generate discrete tones from 1.0 to $3.0 \mathrm{kHz}$ with sound pressure levels of $130 \mathrm{~dB}$ at the liner leading edge. A specially designed plenum allows flow to be combined with the sound field such that sound transmission efficiency is maximized. The uniform flow Mach number used to educe each impedance in this report was taken to be the average value of the Mach number profile measured at the mid-liner axial plane (406 mm downstream of the source plane). Tests were conducted for centerline Mach numbers of $0.0,0.1,0.3$ and 0.5 .

Acoustic waves are propagated from left to right in figure 3 , across the surface of the test liner, and into a termination section designed to minimize reflections over the frequency range of interest. Two $6.35 \mathrm{~mm}$ (1/4-inch) condenser-type microphones are used to acquire the complex acoustic pressure data. The first is a reference microphone, which is flushmounted in the side wall at the test liner leading edge. The amplitude of the discrete tone supplied by the acoustic drivers was controlled to achieve the desired level at this location. For the current investigation, this level was set to $130 \mathrm{~dB}$. This level was chosen because it provides a reasonable signal-tonoise ratio, yet remains below the regime where nonlinear acoustic propagation typically occurs. The second microphone is flush-mounted in an axial traverse bar, which forms a portion of the upper wall of the test section. A $13 \mathrm{~mm}$-wide precision-machined slot in the top wall of the grazing incidence tube allows this axial traverse bar to traverse the test section length by means of a computer-controlled digital stepping motor. The data acquisition program automatically positions the traversing microphone at pre-selected locations, $x_{i}$, from $203 \mathrm{~mm}$ upstream of the leading edge to $203 \mathrm{~mm}$ downstream of the trailing edge of the liner. At each measurement location, a transfer function between the traversing and reference microphones is used to determine the sound pressure level $\operatorname{SPL}\left(x_{i}\right)$ and phase $\phi\left(x_{i}\right)$ relative to the fixed reference microphone location. The complex acoustic pressure at a given axial wall location is determined from the equation

$$
p\left(x_{i}, H\right)=p_{\text {ref }} 10^{\mathrm{SPL}\left(x_{i}\right) / 20} e^{i \phi\left(x_{i}\right)}
$$

where the reference pressure, $p_{\text {ref }}$, is $20 \mu \mathrm{Pa}$.

The GIT was designed for operation with source frequencies below the cut-on frequency of higherorder modes. However, higher-order mode effects cannot be avoided in the liner region. These higherorder modes, as well as reflections, are also generally present in the vicinity of the leading and trailing edges of the liner. For this reason, the source frequency was kept below the cut-on frequency of higher-order hardwall modes. The sole exception to this was the case of $3 \mathrm{kHz}$ at Mach 0.5 . At this frequency and Mach number, the first higher-order mode is capable of carrying energy. However, previous tests have indicated that the plane-wave mode remains dominant for this condition. Higher-order mode effects caused by the installation of the test liner are expected to decay upstream of the leading edge of the test liner.

Higher-order mode effects decay downstream of the liner as well. Thus, the exit plane region (203 $\mathrm{mm}$ downstream of the liner trailing edge) is assumed to contain only plane waves. In order to determine the acoustic impedance at the exit plane, the two complex acoustic pressures that are measured with the traversing bar microphone at the two farthest downstream locations are used. The twomicrophone method is applied as described earlier, except the traverse of the measurement microphone eliminates the need for a "switching operation." The instrumentation used for the GIT is identical to that for the NIT.

\section{$\underline{\text { Analysis }}$}

The applicable geometry and coordinate system used to model the flow-duct test section for the finite element method (FEM) are depicted in figure 4. This method is described in detail elsewhere, ${ }^{1}$ therefore, only sufficient detail is presented here for completeness. The assumption is made that the mean flow profile is uniform and only plane acoustic waves exist in the spanwise direction (perpendicular to the $x-y$ plane; not shown in the sketch). The maximum frequency $(3.0 \mathrm{kHz})$ is below the cut-on frequency for higher-order modes in a hardwall region for all but the highest Mach number $(\mathrm{M}=0.5)$ tested. In the lined section, the two side walls are hard; thus, 


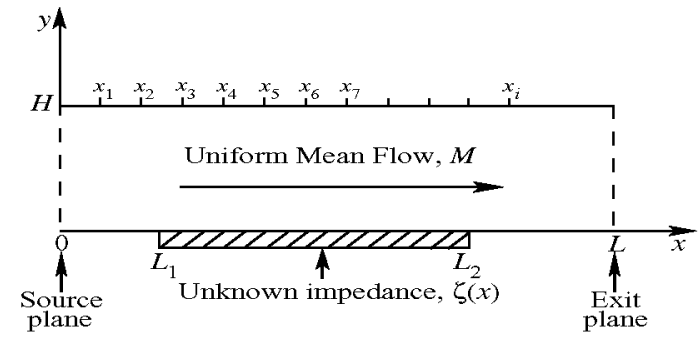

Fig. 4. Test section sketch.

the assumption that no spanwise higher-order modes carry energy is reasonable.

The regions upstream $\left(0 \leq x \leq L_{1}\right)$ and downstream $\left(L_{2} \leq x \leq L\right)$ of the liner contain rigid walls. As described earlier, the complex acoustic pressures are measured at each of the measurement locations along the upper wall (at $x=0, x_{1}, x_{2}, \ldots x_{i}$ ) using the microphone flush-mounted in the traversing bar.

The FEM finds the solution to the steady-state form of the convected wave equation,

$$
\left(1-M^{2}\right) \frac{\partial^{2} p}{\partial x^{2}}+\frac{\partial^{2} p}{\partial y^{2}}-2 i k M \frac{\partial p}{\partial x}+k^{2} p=0
$$

The source plane acoustic pressure boundary condition is

$$
p(0, y)=p_{s}(y)
$$

where $p_{s}(y)$ is the measured acoustic pressure profile in the transverse direction at the source plane. Because only plane waves are assumed at the source plane, $p_{s}(y)$ is set to the constant value measured with the traversing microphone placed in the source plane.

The exit plane boundary condition is

$$
\frac{\partial p(L, y)}{\partial x}=\frac{-i k p(L, y)}{M+\zeta_{e}(y)}
$$

which is derived from the requirement that the exit impedance, $\zeta_{e}(y)$, must equal the ratio of the acoustic pressure to the axial acoustic velocity in that plane. Since only plane waves are assumed to be present at the exit plane, $\zeta_{e}$ is taken to be the constant value determined using flush-mounted microphones and plane wave analysis. ${ }^{11}$ The boundary conditions at all rigid walls are given as

$$
\frac{\partial p}{\partial y}=0
$$

which means the normal component of acoustic particle velocity vanishes at a rigid wall. Finally, the boundary condition for the lined region of the duct ( $L_{1} \leq x \leq L_{2}$ in figure 4 ) is given by ${ }^{12}$

$$
\begin{aligned}
\frac{\partial p(x, 0)}{\partial y}= & \frac{i k p(x, 0)}{\zeta(x)}+2 M \frac{\partial}{\partial x}\left[\frac{p(x, 0)}{\zeta(x)}\right] \\
& +\frac{M^{2}}{i k} \frac{\partial^{2}}{\partial x^{2}}\left[\frac{p(x, 0)}{\zeta(x)}\right]
\end{aligned}
$$

(The reader is reminded that an $e^{i \omega t}$ time convention is used throughout this paper.)

Equations (2)-(6) constitute a boundary value problem that can be solved to obtain the upper wall complex acoustic pressure when the impedance of the liner, $\zeta(x)$, is known. Since each of the test liners included in the current investigation were designed with uniform geometry, their respective impedances were assumed to be constant. In other words, the acoustic impedance of a selected test liner was assumed to be "smeared" over the surface of the test liner, such that axial dependence at the scale of the hole separation was eliminated. The following iterative procedure is applied to educe this acoustic impedance:

1. The FEM is used to solve the boundary value problem and to compute the upper wall acoustic pressure profile.

2. These acoustic pressures are compared to those measured with the traversing microphone.

3. If the computed and measured complex acoustic pressures are matched to within an acceptable error limit, the assumed liner impedance is accepted as being correct and the iteration is terminated.

4. If the computed and measured complex acoustic wall pressures are not sufficiently matched, the assumed liner impedance is updated and steps 1 through 4 are repeated.

For the current study, the normalized acoustic resistance and reactance of the test liner were constrained to $0 \leq \theta \leq 10,-10 \leq \chi \leq 10$.

\section{Results and Discussion}

Each of the eight liner configurations was tested with the NASA Langley Research Center normal incidence and grazing incidence tubes. The first two series of tests were conducted to determine the effects of percent open area and perforate sheet thickness-to-hole diameter ratio on acoustic impedance. Next, tests were conducted to assess the effects of perforate sheet thickness on the acoustic impedance of the relevant liners. Finally, two 
liner configurations were tested to demonstrate the effects of cavity depth.

\section{Effect of Percent Open Area}

Liner configurations 1 through 4 were used to assess the effects of percent open area (POA) on the acoustic impedance of single-layer perforate liners. As described in table 1, the hole diameter, sheet thickness and cavity depth were held constant for this set of liner configurations. By varying the number of holes, the percent open area $(P O A)$ was varied from $6.4 \%$ to $15.0 \%$.

The $51 \mathrm{~mm} \times 51 \mathrm{~mm}$ liners for configurations 1 through 4 were tested in the normal incidence tube (NIT). Each liner was successively mounted onto the end of the NIT, and the two-microphone method was used to educe the normal incidence acoustic impedance. Each of these tests was conducted with the discrete-tone source set to $120 \mathrm{~dB}$ at the reference microphone location, such that any nonlinear effects would be due to the acoustic liner and not the acoustic environment. Figure 5 and table 2 contain the normal incidence impedances for each of these liners, at frequencies of 1.0 to $3.0 \mathrm{kHz}$. The acoustic resistance is observed to decrease from 0.23 to 0.15 as the POA is increased from $6.4 \%$ (liner 1 ) to $8.7 \%$ (liner 2). As the $P O A$ is further increased to $13.2 \%$ (liner 3 ), the acoustic resistance decreases to 0.08 . However, the acoustic resistance remains virtually constant as the $P O A$ is increased to $15.0 \%$. Clearly, the acoustic resistance increases with decreasing $P O A$. Similarly, it is apparent from table 2 that the acoustic reactance also increases with decreasing POA. This is due to the increase in mass reactance as the $P O A$ is decreased. This increase in mass reactance causes the resonance (positive-going zero crossing of reactance curve) to shift to a lower frequency.

Next, the $51 \mathrm{~mm} \times 406 \mathrm{~mm}$ liners for configurations 1 through 4 were sequentially tested in the grazing incidence tube (GIT), at centerline Mach numbers of $0.0,0.1,0.3$ and 0.5 . For these tests, the SPL at the source plane was set to $130 \mathrm{~dB}$. This level was chosen such that the signal-to-noise ratio of the attenuated signal at the trailing edge of the liner would be sufficient to achieve repeatable results. Although this SPL is higher than that used in the data presented from the NIT tests, the fact that the Mach 0.0 impedances educed in the GIT are very nearly identical to the NIT results indicates that $130 \mathrm{~dB}$ is still within the linear acoustic wave propagation regime. To conserve space, only the Mach 0.3 and 0.5 results are provided for all test frequencies in figures 6 and 7 . Again, the acoustic

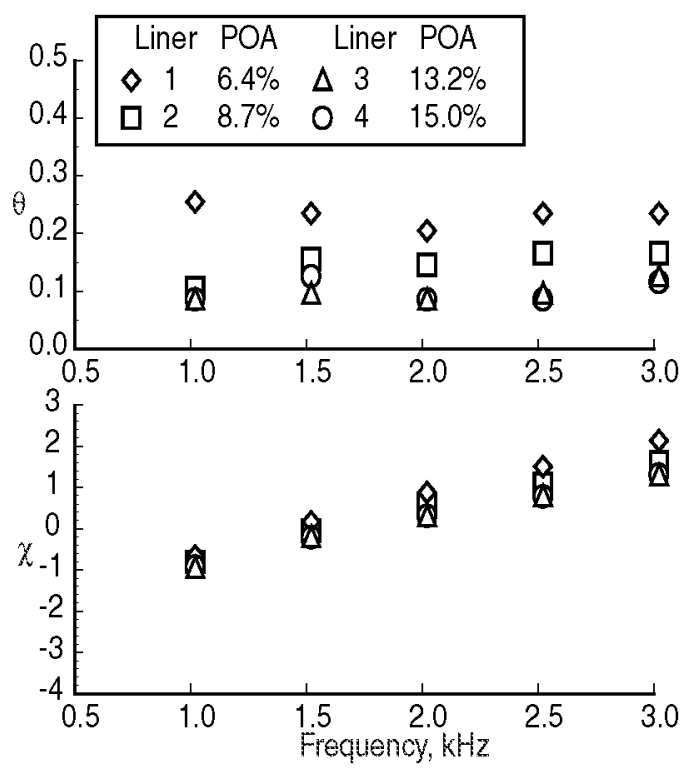

Fig. 5. Effect of $P O A$ on impedance
variation with frequency $(\mathrm{M}=0.0$,
acquired in normal incidence tube).

resistance increases with decreasing $P O A$, and also increases with increasing flow velocity. The acoustic reactance results follow the same trend as seen in the NIT; however, the compressed scale (chosen to allow the comparison of results for all of the liners on identical scales) collapses the symbols such that the trend is not as clearly evident.

Figure 8 provides a comparison of $P O A$ vs $\theta$, for Mach numbers of 0.0,0.1,0.3 and 0.5. The normalized resistances used in this figure are those educed at $2.0 \mathrm{kHz}$, which was the frequency closest to the resonance of each of these four liners. This frequency was chosen such that the cavity effects could be ignored, and the resistance of the perforate sheet would be dominant. As mentioned earlier, the effects of high speed grazing flow for low POA liners have not been well understood. It is useful, therefore, to note that the acoustic resistances for a liner with a $P O A$ of $6.4 \%$ follow the trends measured with larger $P O A$ liners over the Mach number range of 0.0 to 0.5 .

It is also of interest to compare these results with the acoustic resistance model of Rice and Heidelberg, 13 as given by

$$
\theta_{\mathrm{RH}}=\frac{M}{\sigma\left(2+1.256\left(\delta^{*} / d\right)\right)}
$$

where $M$ is the flow Mach number, $\sigma$ is the porosity $(P O A / 100), \delta^{*}$ is the boundary layer displacement 


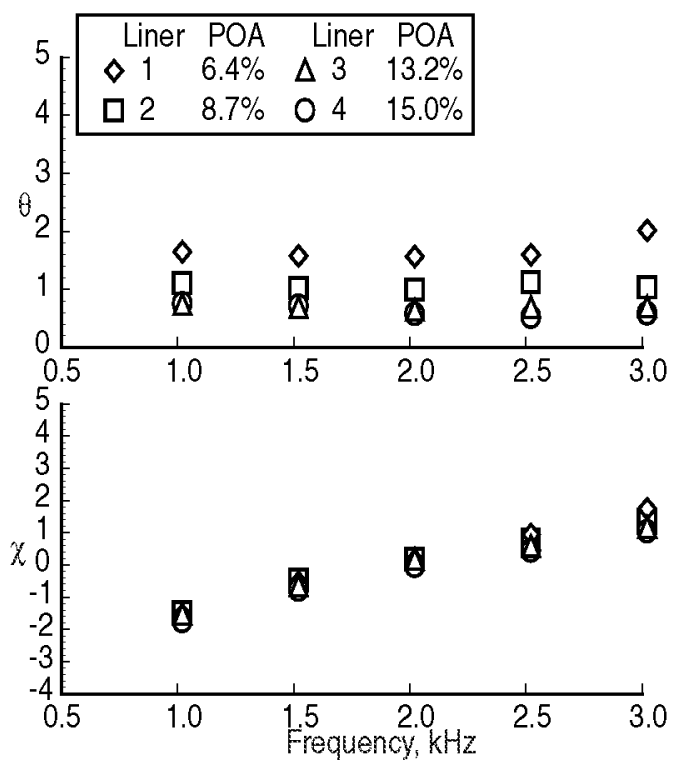

Fig. 6. Effect of $P O A$ on impedance variation with frequency $(\mathrm{M}=0.3)$.

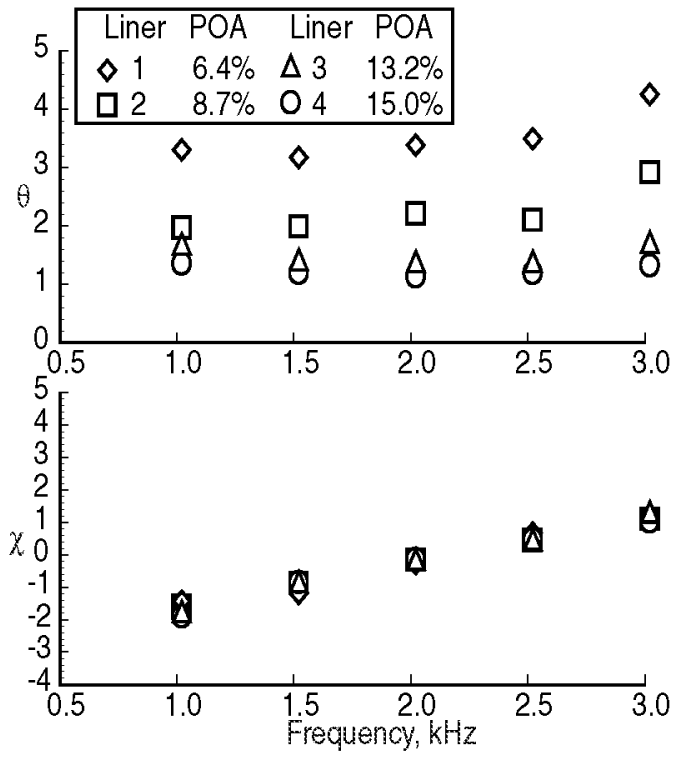

Fig. 7. Effect of $P O A$ on impedance variation with frequency $(M=0.5)$.

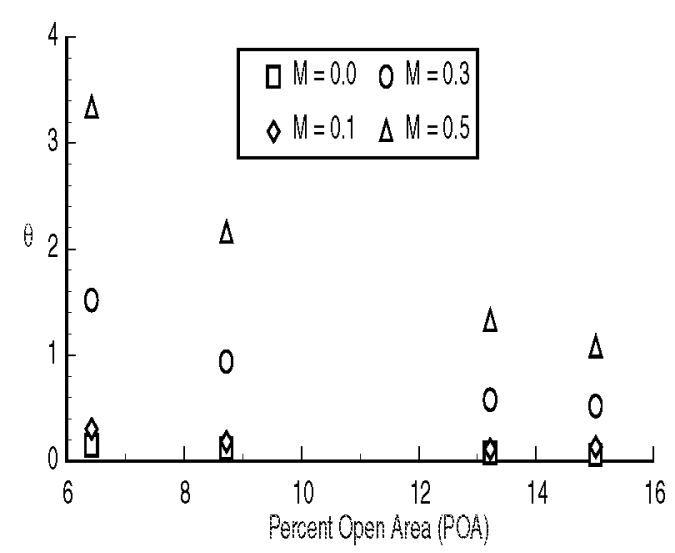

Fig. 8. Effect of $M$ on acoustic resistance variation with $P O A$ ( $M=0.0$ to 0.5$)$.

thickness, and $d$ is the hole diameter. Equation (7) does not include the effects of frequency and perforate sheet thickness, and it does not account for the intrinsic resistance that is present in the absence of flow. Regardless, it is of interest to compare the trends of this approximation with the results acquired in the current investigation at $2 \mathrm{kHz}$ (near resonance). The differences between the Mach 0.3 and Mach 0.5 acoustic resistances predicted using the Rice-Heidelberg model are 0.6, 0.4, 0.3 and 0.2 for POA's of $6.4 \%, 8.7 \%, 13.2 \%$ and $15.0 \%$, respectively. The corresponding educed acoustic resistance differences are 1.8, 1.3, 0.7 and 0.6 at $2 \mathrm{kHz}$. Thus, while the trends are generally similar, the $\theta$ values educed from measured data are observed to be approximately three times the predicted $\theta_{\mathrm{RH}}$ values over the $P O A$ entire range.

\section{Effect of Perforate Sheet Thickness}

Liner configurations 6 and 7 were used to study the effects of perforate sheet thickness on the acoustic impedance. As shown in table 1, the sheet thicknesses were $0.51 \mathrm{~mm}$ and $1.02 \mathrm{~mm}$, while all other dimensions were held constant. Table 2 and figure 9 contain the results acquired with these two liners in the NIT and GIT, respectively. The results at Mach 0.0 are similar; however, the values of $\theta$ and $\chi$ are slightly higher for liner 6 than for liner 7 . The same holds true for the GIT results at Mach 0.3 and 0.5 . Regardless, this difference in thickness does not appear to be sufficient to cause appreciable differences in the acoustic impedance. Apparently, the acoustic resistance viscous component, which is assumed to be proportional to sheet thickness, is being swamped by SPL and mean flow nonlinear effects. 

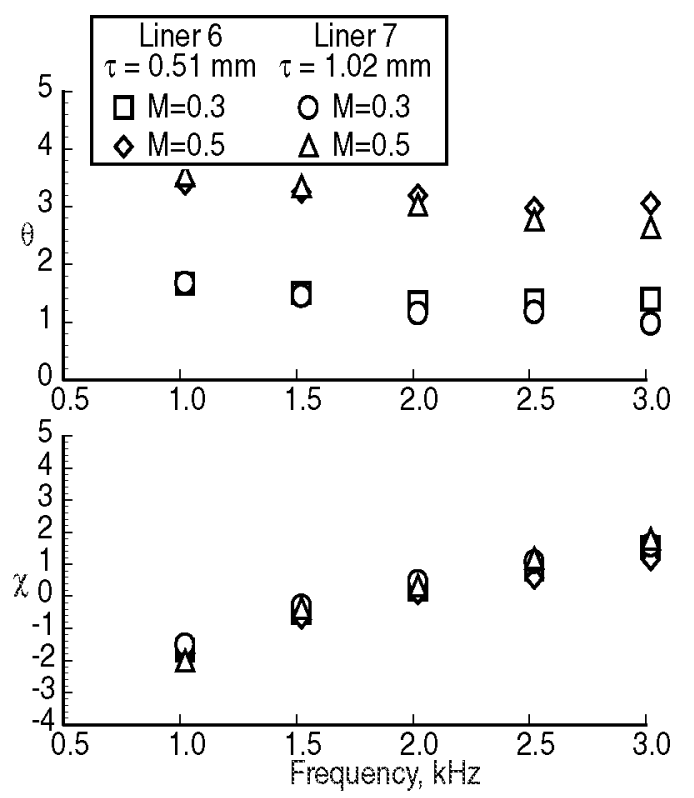

Fig. 9. Effect of perforate sheet thickness on impedance variation with frequency

$(M=0.3$ and 0.5$)$.

\section{Effect of Perforate Sheet Thickness-to-Hole Diameter Ratio}

Liners 3 and 5 were used to assess the effects of sheet thickness-to-hole diameter $(\tau / d)$ ratio on the acoustic impedance of single-layer conventional liners. The $\tau / d$ ratios for liners 3 and 5 were 0.65 and 0.34 , respectively. As seen in table 1, the POA's for these two liners were slightly different; however, this difference ( $13.0 \%$ vs $13.2 \%$ ) was assumed to be small enough for acceptable comparisons. The normal incidence impedances educed in the NIT are provided in table 2 . Their resistances are nearly identical, and the difference between their reactances increase from about 0.1 to 0.4 as the frequency is increased from 1.0 to $3.0 \mathrm{kHz}$.

The resistances educed in the GIT (figure 10) for the two liners remain essentially the same at Mach 0.3. At Mach 0.5, however, the acoustic resistance of liner 5 is observed to be larger than that for liner 3 , while their respective acoustic reactances are nearly identical. It should be noted that the hole diameter increases from $0.99 \mathrm{~mm}$ to $2.36 \mathrm{~mm}$ as $\tau / d$ decreases from 0.65 to 0.34 . Thus, it is possible that the increase in resistance for liner 5 , relative to that of liner 3 , is caused by an increase in the boundary layer thickness due to the increased diameter of the perforate holes. This possibility is intended to be the subject of a future investigation.

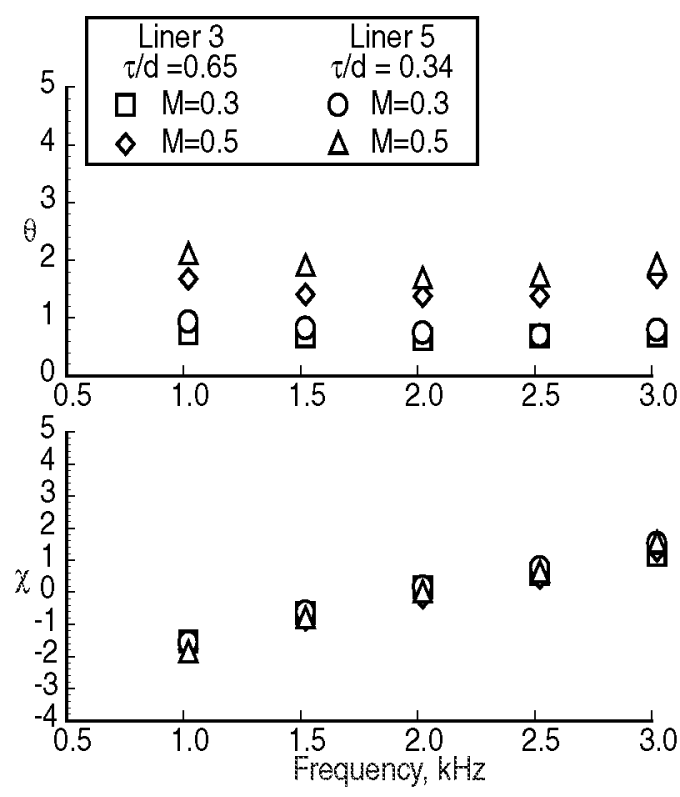

Fig. 10. Effect of $\tau / d$ on impedance variation with frequency ( $M=0.3$ and 0.5$)$.

It should also be noted that the values predicted by the Rice-Heidelberg model $\left(\theta_{\mathrm{RH}}\right)$ increase by 0.7 and 0.9 for liners 3 and 5 , respectively, as the Mach number is increased from 0.3 to 0.5 . The corresponding educed resistances (at $2 \mathrm{kHz}$ ) increase by 0.3 and 0.45 . Thus, the ratio of the predicted vs educed resistance increases, as the Mach number is increased from 0.3 to 0.5 , is nearly constant (2.3 and 2.0 for liners 3 and 5 , respectively).

The two liners (6 and 7) used to assess the $\tau$ effects on liner impedance can also be used to assess $\tau / d$ effects. As shown in table $1, \tau / d$ is 0.40 for liner 6 and 0.80 for liner 7 . Perhaps a comparison of figures 9 and 10 demonstrates one of the effects of $\tau / d$ more clearly. In each figure, $\tau / d$ is observed to be approximately doubled for one liner relative to the other. For a POA of $13 \%$ (liners 3 and 5), the increased $\tau / d$ appears to have a "linearizing" effect, such that the effects of mean grazing flow are reduced. Conversely, for a POA of $8.7 \%$ (liner 6 and 7 ), this "linearizing" effect is not as readily apparent. Evidently, "linearization" cannot be accomplished solely be increasing $\tau / d$.

\section{Effect of Cavity Depth}

The last comparison was for the effects of cavity depth on liner impedance. For this comparison, liner configurations 2 and 8 were used. Liner 2 had a cavity depth of $38.1 \mathrm{~mm}$, while liner 8 had a cavity depth of $76.2 \mathrm{~mm}$. All other dimensions 


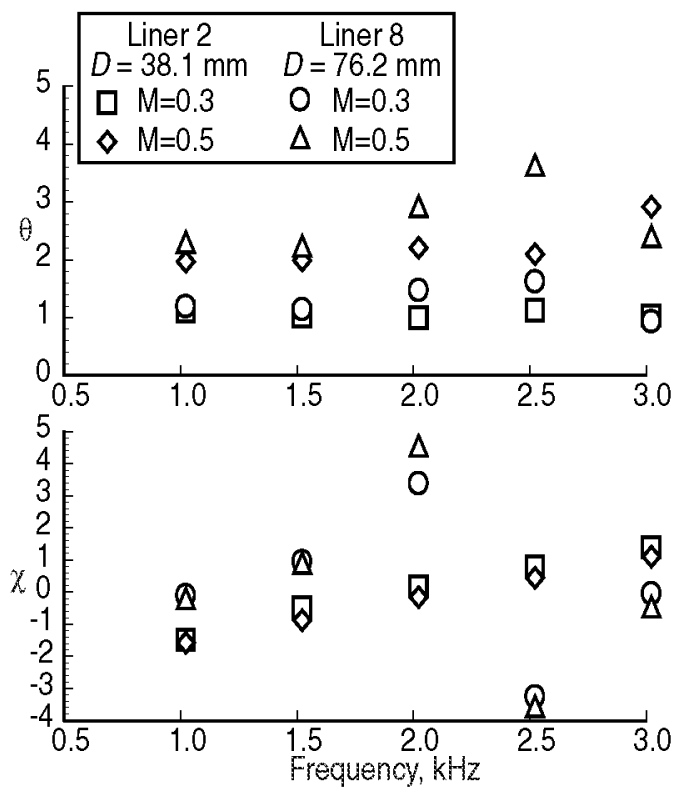

Fig. 11. Effect of cavity depth on impedance variation with frequency ( $M=0.3$ and 0.5$)$.

were maintained constant for these two configurations. The results for these liners are provided in table 2 and figure 11. As expected, the main difference between the results for these two liners is the shift of the resonance frequency. The increase in cavity depth moves the anti-resonance frequency from approximately $4.0 \mathrm{kHz}$ (outside the frequency range of interest for this investigation) to approximately $2.0 \mathrm{kHz}$. Thus, the strong anti-resonance behavior of the reactance becomes readily apparent for the liner with a larger cavity depth. Also, the acoustic resistances near the $2 \mathrm{kHz}$ anti-resonance for liner 8 are increased by approximately 0.2 over the resistance for liner 2 at this frequency. The results educed in the GIT, at Mach numbers of 0.3 and 0.5 , contain the same trends seen in the NIT data, with the variability in the vicinity of the anti-resonance significantly enhanced. At frequencies away from antiresonance, the acoustic resistance is virtually unaffected by changes in the cavity depth. Overall, the resonance frequency dependence on cavity depth is much stronger than any other cavity depth effect.

\section{Concluding Remarks}

A series of tests was conducted with selected liner configurations in the NASA Langley Flow Impedance Test Facility, using a normal incidence tube and a grazing incidence tube. These configurations were selected to demonstrate the effects of percent open area, sheet thickness, sheet thicknessto-hole diameter ratio and cavity depth in perforate liners. The two-microphone method was used to educe normal incidence acoustic impedances from the normal incidence tube data, and a finite element method was used to educe the impedances in the presence of grazing flow from the grazing incidence tube data. The results of this study provide a quantitative assessment of the effects of liner geometry on the acoustic impedance of perforate, single-layer acoustic liners currently used in commercial aircraft. Of the parameters (and their respective ranges) included in this study, the relative order of importance is (1) percent open area $(P O A),(2)$ sheet thicknessto-hole diameter ratio $(\tau / d),(3)$ cavity diameter $(D)$ and (4) sheet thickness $(\tau)$. However, it is important to note the strong interactions between the different parameters. In real applications, it is sometimes too expensive to fabricate liners with ideal geometry. In these cases, trade-offs between the different parameters must be made. This report offers additional data for use in these trade-offs.

Key observations regarding effects of liner geometry on acoustic impedance, for the parameter ranges used in the current investigation $(6.4 \% \leq P O A \leq$ $13.2 \%, 0.34 \leq \tau / d \leq 0.80,0.51 \mathrm{~mm} \leq \tau \leq 1.02 \mathrm{~mm}$, $38.1 \mathrm{~mm} \leq D \leq 76.2 \mathrm{~mm}$ ) are:

1. Both resistance and reactance increase with decreasing $P O A$. This behavior becomes increasingly significant for the resistance component as the Mach number is increased from 0.0 to 0.5 , and follows trends predicted by current models.

2. At Mach numbers up to 0.3 , the acoustic impedance is not affected by changes in $\tau / d$ over the range considered in the current study. At Mach 0.5 , the reactance remains virtually unaffected by changes in $\tau / d$. However, at $P O A=13 \%$, the resistance is "linearized" by the increase in $\tau / d$, such that increases in the resistance due to increases in $M$ are less for the higher $\tau / d$ value. On the other hand, at $P O A=8.7 \%$, this "linearization" due to increased $\tau / d$ is not readily apparent. More importantly, the ratio of resistance increases predicted by the Rice-Heidelberg model to those educed in the current study, as the Mach number increases from 0.3 to 0.5 , is nearly constant.

3. The resonance frequency decreases as $D$ increases. Also, the acoustic resistance increases in the vicinity of the anti-resonance frequency.

4. Liner impedance was independent of $\tau$ for the cases included in this study. 


\section{$\underline{\text { References }}$}

${ }^{1}$ Watson, W. R., Jones, M. G., and Parrott, T. L., "Validation of an Impedance Eduction Method in Flow," AIAA Journal, Vol. 37, No. 7, 1999, pp. 818824 .

2Ingard, U., "On The Theory And Design Of Acoustic Resonators," Journal of Acoustical Society of America, Vol. 25, No. 6, 1953, pp. 1037-1061.

${ }^{3}$ Rice, E.J., "A Model For The Acoustic Impedance Of A Perforated Plate Liner With Multiple Frequency Excitation." NASA TMX-67950, 1971.

${ }^{4}$ Melling, T.H., "The Acoustic Impedance of Perforates at Medium and High Sound Pressure Levels." Journal of Sound and Vibration, Vol. 29, No. 1, 1973, pp. 1-65.

${ }^{5}$ Guess, A.W., "Calculation Of Perforated Plate Liner Parameters From Specified Acoustic Resistance And Reactance," Journal of Sound and Vibration, Vol. 40, No. 1, 1975, pp. 119-137.

${ }^{6}$ Hersh, A.S., Walker, B., and Bucka, M., "Effect Of Grazing Flow On The Acoustic Impedance Of Helmholtz Resonators Consisting Of Single And Clustered Orifices." AIAA 78-1124, 1978, pp. 1-14.

7 "Aeroacoustics of Flight Vehicles: Theory and Practice - Volume 1: Noise Sources," NASA RP1258, edited by H.H. Hubbard, 1991.

${ }^{8}$ Parrott, T. L. and Jones, M. G., "Parallelelement Liner Impedances for Improved Absorption of Broadband Sound in Ducts," Noise Control Engineering Journal, 1995, pp. 183-195.

${ }^{9}$ Premo, J., "The Application Of A Time-Domain Model To Investigate The Impedance Of Perforate Liners Including The Effects Of Bias Flow," $A I A A$ Paper 99-1876, 1999.

10 Armstrong, D. L., Beckemeyer, R. J. and Olsen, R. F., "Impedance Measurements of Acoustic Duct Liners With Grazing Flow," Paper presented at the $87^{\text {th }}$ Meeting of the Acoustical Society of America, New York, NY, 1974.

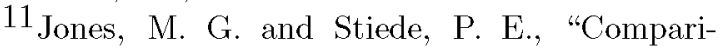
son of Methods for Determining Specific Acoustic Impedance," Journal of Acoustical Society of A merica, Vol. 101, No. 5, 1997, pp. 2694-2704.

12 Myers, M. K., "On the Acoustic Boundary Condition in the Presence Of Flow," Journal Of Sound And Vibration, Vol.'71, No.'3, 1980, pp. 429-434.

${ }^{13}$ Heidelberg, L.J., Rice, E.J. and Homyak, L., "Experimental Evaluation of a Spinning-Mode Acoustic Treatment Design Concept for Aircraft Inlets, NASA TP-1613, 1980
Table 1 - Acoustic Liner Configurations

\begin{tabular}{|c|c|c|c|c|c|}
\hline $\begin{array}{c}\text { Config } \\
(\#)\end{array}$ & $\begin{array}{c}\text { POA } \\
(\%)\end{array}$ & $\begin{array}{c}d \\
(\mathrm{~mm})\end{array}$ & $\begin{array}{c}\tau \\
(\mathrm{mm})\end{array}$ & $\tau / d$ & $\begin{array}{c}D \\
(\mathrm{~mm})\end{array}$ \\
\hline 1 & 6.4 & 0.99 & 0.64 & 0.65 & 38.1 \\
\hline 2 & 8.7 & 0.99 & 0.64 & 0.65 & 38.1 \\
\hline 3 & 13.2 & 0.99 & 0.64 & 0.65 & 38.1 \\
\hline 4 & 15.0 & 0.99 & 0.64 & 0.65 & 38.1 \\
\hline 5 & 13.0 & 2.36 & 0.81 & 0.34 & 38.1 \\
\hline 6 & 7.3 & 1.27 & 0.51 & 0.40 & 38.1 \\
\hline 7 & 7.3 & 1.27 & 1.02 & 0.80 & 38.1 \\
\hline 8 & 8.7 & 0.99 & 0.64 & 0.65 & 76.2 \\
\hline
\end{tabular}

Table 2 - Normal Incidence Impedances using NIT (Total SPL of $120 \mathrm{~dB}$ - Tone)

(a) Acoustic Resistance, $\theta$

\begin{tabular}{|c|c|c|c|c|c|}
\cline { 2 - 6 } \multicolumn{1}{c|}{} & \multicolumn{5}{c|}{ Frequency, $\mathrm{kHz}$} \\
\hline Liner \# & 1.0 & 1.5 & 2.0 & 2.5 & 3.0 \\
\hline 1 & 0.25 & 0.23 & 0.20 & 0.23 & 0.23 \\
\hline 2 & 0.10 & 0.15 & 0.14 & 0.16 & 0.16 \\
\hline 3 & 0.08 & 0.09 & 0.08 & 0.09 & 0.12 \\
\hline 4 & 0.08 & 0.12 & 0.08 & 0.08 & 0.11 \\
\hline 5 & 0.08 & 0.11 & 0.08 & 0.09 & 0.11 \\
\hline 6 & 0.10 & 0.16 & 0.12 & 0.15 & 0.15 \\
\hline 7 & 0.12 & 0.15 & 0.19 & 0.24 & 0.23 \\
\hline 8 & 0.12 & 0.14 & 0.41 & 0.38 & 0.18 \\
\hline
\end{tabular}

(b) Acoustic Reactance, $\chi$

\begin{tabular}{|c|r|r|c|c|c|}
\cline { 2 - 6 } \multicolumn{1}{c|}{} & \multicolumn{5}{c|}{ Frequency, $\mathrm{kHz}$} \\
\hline Liner \# & 1.0 & 1.5 & 2.0 & 2.5 & 3.0 \\
\hline 1 & -0.73 & 0.10 & 0.81 & 1.44 & 2.07 \\
\hline 2 & -0.89 & -0.13 & 0.48 & 1.00 & 1.53 \\
\hline 3 & -1.02 & -0.28 & 0.23 & 0.71 & 1.22 \\
\hline 4 & -1.00 & -0.29 & 0.23 & 0.70 & 1.23 \\
\hline 5 & -0.87 & -0.07 & 0.50 & 1.03 & 1.64 \\
\hline 6 & -0.78 & -0.02 & 0.63 & 1.21 & 1.80 \\
\hline 7 & -0.66 & 0.23 & 0.93 & 1.49 & 2.18 \\
\hline 8 & 0.16 & 1.12 & 3.64 & -1.95 & 0.38 \\
\hline
\end{tabular}

